\title{
舰船空气流场数值模拟及特性分析
}

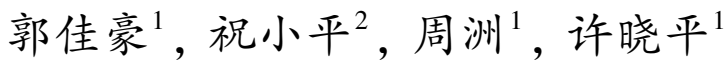

(1.西北工业大学 航空学院，陕西 西安 710072；2.西北工业大学 无人机特种技术重点实验室，陕西 西安 710065)

\begin{abstract}
摘 要: 应用计算流体力学技术对舰船空气流场进行数值模拟, 对比了求解雷诺平均 Navier-Stokes 方 程(RANS) 与分离涡模拟(DES) 的结果, 总结了 2 种方法在舰船流场模拟中的特点。结果表明,舰船 空气流场呈现出非稳态特征, 基于 RANS 的数值模拟精度不足, 而 DES 方法能够较为准确地捕捉流场 中涡的生成和脱落,且与实验符合较好。接着采用 DES 方法对航母空气流场进行模拟,对比了不同风 向下流场中的涡流特征及其对舰载机进舰轨迹上速度分布的影响。发现航母舰岛、甲板等钝体边缘 产生相互千扰的复杂涡系, 其导致舰载机进舰轨迹上产生强烈的速度波动,这种波动主要集中在航母 后部甲板及邻近的尾流区，且随着时间的推进而变化，呈现一定的周期性。
\end{abstract}

关 键 词: 舰船尾流; 数值方法;DES; 航母; 涡

中图分类号:U661.1 文献标志码:A

舰船上复杂的气流直接影响舰载飞行器的正常 起降, 导致驾驶难度增加, 严重时甚至威胁到驾驶员 的生命, 因此对舰船空气流场特性的研究有着重要 意义。

风洞实验是舰船空气流场特性研究的有效手 段。Zan 等 ${ }^{[1-2]}$ 通过油膜成像法及热线风速仪分别 对简单护卫舰 SFS 1:60 缩比模型与 SFS2 1：100 缩比模型的流场进行了测量。Rajagopalan 等 ${ }^{[3]}$ 采 用粒子图像测速法 (particle image velocimetry, PIV) 测量了两栖攻击舰 LHA 1:48 缩比模型在不同风 速和不同风向下的流场。国内方面, 刘航等 ${ }^{[4]}$ 在风 洞中观测到了舰船飞行甲板上方流动强烈的随机 性。赵维义等 ${ }^{[5-6]}$ 均开展了舰船空气流场的 PIV 测 量实验。但风洞实验存在周期长、成本高的缺点。

随着计算流体力学 (CFD) 技术的发展, 数值模 拟方法已广泛应用于舰船流场计算之中。Reddy 等 ${ }^{[7]}$ 基于结构网格定常计算, 分析了简单护卫舰模 型甲板上方不同位置的速度分布。Polsky 等 ${ }^{[8]}$ 对 LHA 进行了数值模拟, 发现非定常取得的时均结果 与实验数据更加符合, 且舰船流场结构表现出雷诺 数无关性。陆超等 ${ }^{[9-10]}$ 通过对 LHA 缩比模型进行 数值模拟, 探讨了相似准数对舰面空气流场的影响,
文章编号: 1000-2758(2018) 06-1037-08

并对比了 2 种不同飞行甲板的流场特征。贲亮亮 等 ${ }^{[11]}$ 通过求解定常雷诺平均 Navier-Stokes 方程 (RANS), 分析了不同风向和风速下舰载机进舰轨 迹上的速度分布特征, 但由于舰船流场体现出非稳 态特征, 速度分布随着时间推进会产生较大的波动, 定常计算并不能得到这种速度变化。郜冶等 ${ }^{[12-14]}$ 则通过求解非定常雷诺平均 Navier-Stokes 方程 (URANS) 对不同风向下甲板浴结构进行了分析, 并 模拟了舰载机滑跃起飞过程中舰面流场变化, 但其 在验证数值方法时与实验数据对比仍有较大差距。 上述研究大多采用 RANS 求解, 但局限于 RANS 自 身的数值耗散, 对舰船流场非定常现象模拟及浴结 构捕捉的精度有待进一步提高。而 Forrest ${ }^{[15]}$ 和操 戈等 ${ }^{[16]}$ 通过模拟 SFS2 的流场, 验证了分离涡模拟 (detached eddy simulation, DES) 捕捉舰船流场浴结 构的能力。

本文将分别采用 RANS 与 DES 方法, 对比其在 舰船空气流场研究中的特点。并通过 DES 数值模 拟方法分析航母空气流场的主要特征及复杂流动在 舰载机进舰轨迹上引起的速度波动, 以此探究舰载 机的着舰环境。 


\section{DES 模型简介}

DES 方法是将 RANS 与大浴模拟 ( large eddy simulation, LES) 结合起来进行求解的一种混合方 法 ${ }^{[17-18]}$ 。其将 RANS 湍流模型中的长度尺度 $l_{\mathrm{R}}$ 与 LES 长度尺度 $l_{\mathrm{L}}$ 用新的长度尺度 $l_{\mathrm{D}}$ 替换:

$$
l_{\mathrm{D}}=\min \left(l_{\mathrm{R}}, l_{\mathrm{L}}\right)
$$

对于 S-A 湍流模型, 长度尺度即距壁面的距离:

$$
l_{\mathrm{R}}=d
$$

$k-\omega \mathrm{SST}$ 湍流模型的长度尺度为:

$$
l_{\mathrm{R}}=\sqrt{k} / \beta^{*} \omega
$$

$\beta^{*}$ 为 SST 模型中的一个常数, $k$ 与 $\omega$ 分别为湍 动能及湍流比耗散率。LES 长度尺度为:

$$
l_{\mathrm{L}}=C_{\mathrm{DES}} \Delta
$$

式中, $\Delta$ 为局部网格尺度, 是网格单元的三维尺寸的 最大值; $C_{\mathrm{DES}}$ 为 DES 模型中的校准常数, 取值为 0.65 。从 $l_{\mathrm{D}}$ 的构造中可以看出, DES 模型在近壁面 区域采用传统 RANS 求解高频小尺度运动, 在其余 区域采用 LES 求解低频大尺度运动。

由于网格疏密程度的不同, LES 计算可能在边 界层中被激活, 但 LES 求解边界层时需要相比 RANS 更大的网格密度。此时若被激活会导致求解 中产生过小的湍流黏性, 从而导致模化应力损耗 ( modeled stress depletion, MSD) 的发生, 致使网格诱 导分离 ${ }^{[19]}$ 。

为解决该问题, DES 方法增加了避免在边界层 中激活 LES 的延迟选项 ${ }^{[19]}$, 采用新的长度尺度 $l_{\mathrm{N}}$ :

$$
l_{\mathrm{N}}=l_{\mathrm{R}}-f_{d} \max \left(0, l_{\mathrm{R}}-C_{\mathrm{DES}} \Delta\right)
$$

$f_{d}$ 为延迟函数:

$$
\begin{gathered}
f_{d}=1-\tanh \left(\left[8 r_{d}\right]^{3}\right) \\
r_{d}=\left(v_{t}+v\right) /\left(\sqrt{\boldsymbol{u}_{i, j} \boldsymbol{u}_{i, j}} \boldsymbol{\kappa}^{2} d^{2}\right)
\end{gathered}
$$

式中, $v_{t}$ 为动浴黏性, $v$ 为分子黏性, $\boldsymbol{u}_{i, j}$ 为速度梯度, $\kappa$ 为 Kármán 常数, $d$ 为距壁面距离。当 $f_{d}$ 接近 0 时 采用 RANS 求解, $f_{d}$ 接近 1 时变为传统 DES 求解方 法。本文采用增加延迟选项的 DES 方法。

\section{2 几何模型及计算条件}

\section{1 计算模型与网格划分}

采用简单护卫舰 SFS2 模型,该模型由标准的几 何体组成,如图 1 所示。其船长 $l=138.684 \mathrm{~m}$, 船宽 $b=13.716 \mathrm{~m}$, 机库高 $h=6.096 \mathrm{~m}$ 。计算采用结构网 格。由于 DES 求解中局部网格尺度 $\Delta$ 取决于网格 单元三维最大尺寸, 因此对船体及周边区域网格划 分时需保证 3 个方向网格尺度的统一。参考文献 $[15,20]$, 保证舰船周围区域的目标网格尺度 $\left(\Delta_{0}\right)$ 为: $\Delta_{0} / b=0.05$ 。对甲板上方这样的 “重点关注区 域”, 网格加密处理, 取 $\Delta_{0} / b=0.03$, 如图 2 所示。调 整第一层网格距离, 保证 $y^{+} \approx 1$, 最终生成网格单元 总量为 486 万。

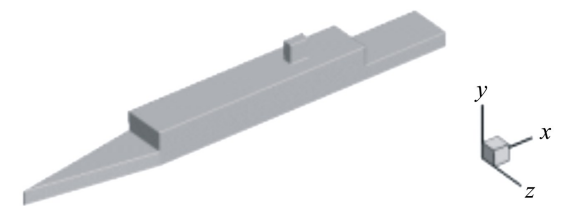

图 $1 \mathrm{SFS} 2$ 几何模型

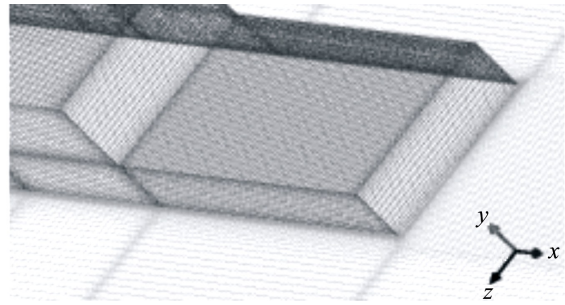

图 2 SFS2 网格局部示意

\section{2 计算条件}

计算采用理想气体模型,计算高度为海平面, 自 由来流速度 $V_{0}=20 \mathrm{~m} / \mathrm{s}$, 来流方向沿舰䑶 ( $x$ 轴) 无 偏转。船体采用无滑移边界条件,计算域下表面选 用零剪切应力壁面 (与风洞实验条件相对应 ${ }^{[15]}$ ), 其余边界设置为压力远场。

\section{3 数值方法验证}

分别采用 RANS 和 DES 方法对 SFS2 全尺寸模 型进行非定常计算。时间步长采用来流速度及船宽 进行无量纲化:

$$
\Delta t^{*}=\Delta t V_{0} / b
$$

式中, $\Delta t$ 为实际时间步长。参考文献 $[15,16,19]$ 中采用的时间步长, 选取 $\Delta t^{*}=2.9 \times 10^{-2}$, 子迭代步 数取 10 。计算基于定常模拟结果,在 500 个时间步 之后对每个时间步的结果进行采样平均, 共采样 1000 个时间步, 最终将得到时均结果与文献 [15] 中实验数据进行对比。 


\subsection{URANS 数值模拟}

采用 $k-\omega \mathrm{SST}$ 湍流模型进行定常与非定常模 拟。在飞行甲板长度 $50 \%$ 、高度与机库平齐处取一 条沿船宽的直线, 读取该直线上的速度, 并与实验数 据进行对比。横坐标采用船宽进行无量纲化, 纵坐 标采用来流速度 $V_{0}$ 无量纲化, 结果如图 3 所示。

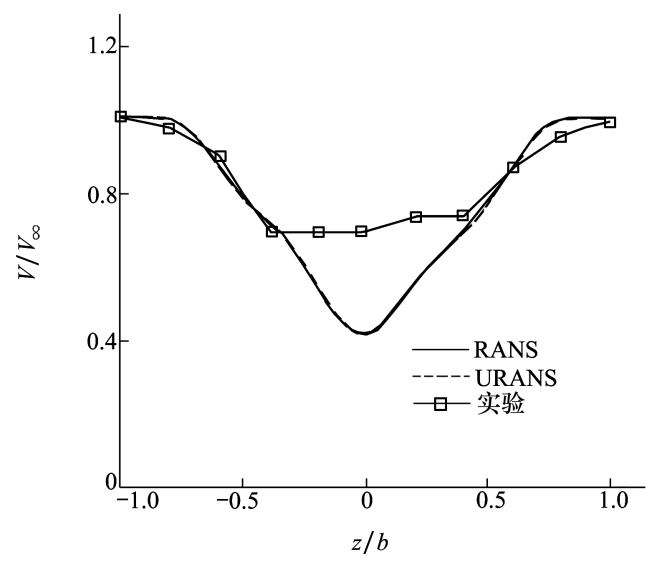

图 3 不同方法 $\mathrm{V}_{\mathrm{x}}$ 速度对比

$k-\omega \mathrm{SST}$ 湍流模型非定常计算所得时均速度分 量 $V_{x}$ 在船中轴附近同实验结果差距较大, 而同定常 计算结果基本一致。这是由于其计算并未捕捉到舰 船流场的非定常流动特征, 计算过程中阻力几乎没 有波动,也说明了这一点。图 4 为定常计算 $z=0 \mathrm{~m}$ 截面的速度云图, 而非定常计算所得瞬态流场与其 结果基本一致, 这里便不再给出。因此, 基于 RANS 的数值模拟对舰船空气流场的计算精度有待进一步 提高。

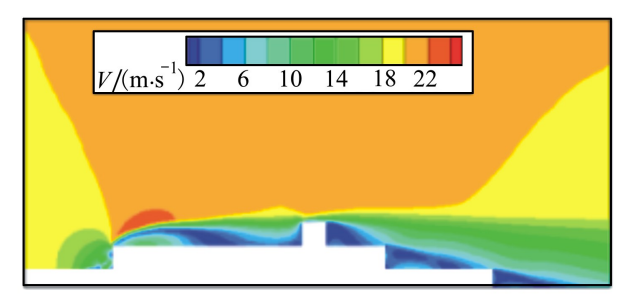

图 4 RANS 定常计算所得 $z=0 \mathrm{~m}$ 截面速度云图

\subsection{DES 数值模拟}

采用基于 S-A 和 $k-\omega$ SST 湍流模型的 2 种 DES 方法进行模拟。从图 5 展示的速度对比中可以看 出, 基于 $k-\omega$ SST 湍流模型的 DES-SST 方法得到的 结果更加精确, 其得到的瞬态速度云图显示出了舰 船流场的非稳态特征, 而时均结果与图 4 中 RANS
定常计算所得的流场结构基本相似,如图 6 所示。
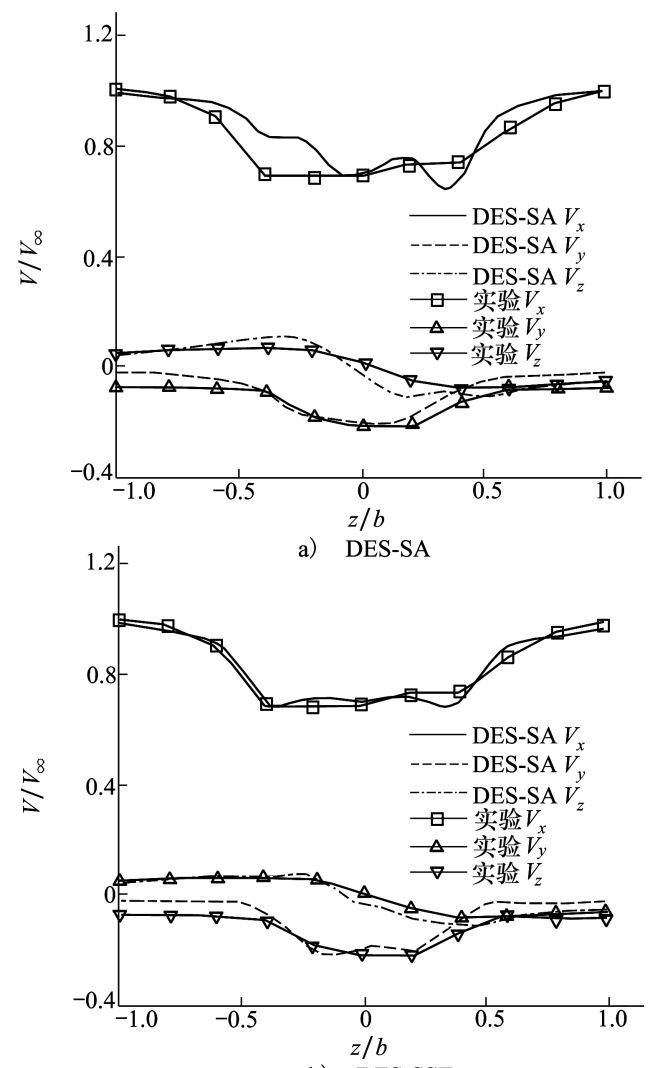

b) DES-SST

图 52 种 DES 计算结果速度分量对比

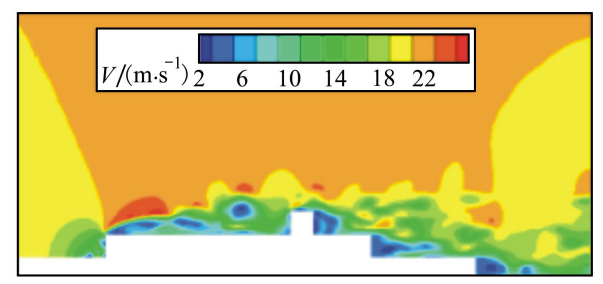

a) 瞬态结果

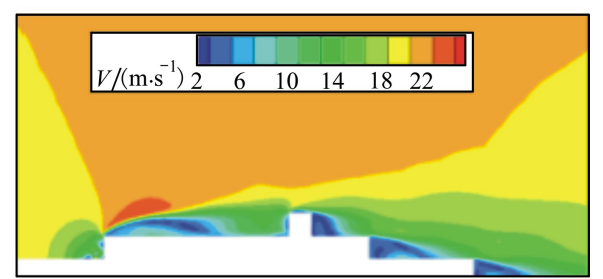

b) 时均结果

图 $6 z=0 \mathrm{~m}$ 截面速度云图 (DES-SST)

\section{4 航母空气流场数值模拟}

通过以上计算可知, 由于 RANS 求解存在数值 耗散, 对旋涡主导的非定常流动具有阻尼抑制作用, 不能捕捉到舰船流场的非稳态特征, 而 DES 方法对 
这类高雷诺数大分离流动的处理有着较好的精度。 因此采用 DES 方法对航母空气流场进行模拟。

\section{1 几何模型与网格生成}

计算选用简化航母模型, 模型甲板长 $l_{0}=260$ $\mathrm{m}$, 宽 $b_{0}=60 \mathrm{~m}$, 甲板高 $h_{0}=17 \mathrm{~m}$, 采用斜角甲板, 倾 斜角为 $9^{\circ}$, 舰载机的理想进舰轨迹正对斜角甲板中 部, 下滑角为 $4^{\circ}$ 。选择第 2 根和第 3 根阻拦索之间, 距甲板 $3 \mathrm{~m}$ 高的位置为理想着舰点, 如图 7 所示。

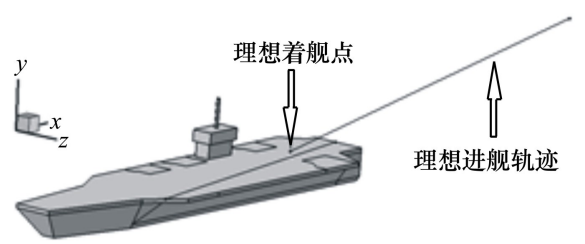

图 7 航母简化模型及理想进舰轨迹示意图

采用结构化网格对整个计算域进行网格划分。 参考 SFS2 算例验证, 对航母周边及尾流区进行加 密, 选取网格尺度 $\Delta_{0} / b_{0}=0.05$, 对舰岛后部甲板区 域选取 $\Delta_{0} / b_{0}=0.03$ 。调整第一层网格距离保证 $y^{+}$ $\approx 1$,生成网格单元总量为 780 万。

\section{2 计算条件}

为模拟航母在真实海面上的流场特征, 考虑海 面上风的影响, 参考文献 [21] 对来流速度进行 建模:

$$
V=V_{\text {ref }}\left(y / y_{\text {ref }}\right)^{\alpha}
$$

式中, $y_{\text {ref }}, V_{\text {ref }}$ 为参考高度及参考高度对应的速度, 对于海平面常数 $\alpha$ 建议取为 $0.13^{[21]}$, 此外取 $V_{\text {ref }}=$ $21.8 \mathrm{~m} / \mathrm{s}, y_{\mathrm{ref}}=300 \mathrm{~m}$, 使甲板高度对应的风速为 $V=$ $15 \mathrm{~m} / \mathrm{s}$ 。忽略航母的六自由度运动, 对平行于海平 面 $0^{\circ}$ 、左船 $9^{\circ}$ 和右船 $15^{\circ}$, 这 3 个风向的空气流场进 行模拟。计算采用 DES-SST 模型, 取无量纲时间步 长 $\Delta t^{*}=2 \times 10^{-2}$,其余计算条件同上。

\section{3 三维流场结构分析}

为分析流场的涡结构, 采用 $Q$ 准则 ${ }^{[22]}$ 对涡核进 行判断。 $Q$ 值的定义为:

$$
Q=1 / 2\left(\|\boldsymbol{\Omega}\|^{2}-\|\boldsymbol{S}\|^{2}\right)
$$

式中, $\boldsymbol{\Omega}$ 和 $\boldsymbol{S}$ 分别为速度梯度张量矩阵的反对称和 对称部分, 涡核存在于 $Q>0$ 的区域。 $Q$ 准则反映 了流场中流体微元旋转和变形之间的平衡, 定义了 旋转占主导地位的浴核区域。

分析 $0^{\circ}$ 、左舷 $9^{\circ}$ 和右舷 $15^{\circ}$, 这 3 个风向的甲板 流场浴结构, 如图 8 所示。从图中可以看出, 航母流
场中的浴流主要在甲板、舰岛等钝体的边缘产生, 主 要有: 舰艄处的分离涡、舰岛处周期脱落的浴、尾部 甲板后方由于陡壁效应形成的涡以及甲板两侧卷起 的涡。

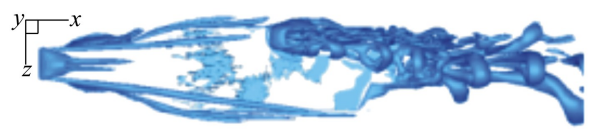

a) $0^{\circ}$ 风向

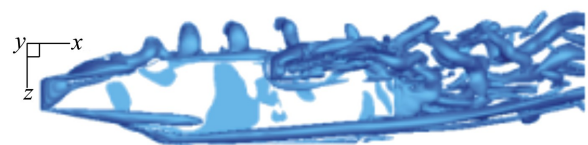

b）左舷 $9^{\circ}$ 风向

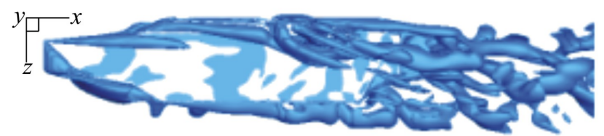

c）右舷 $15^{\circ}$ 风向

图 8 不同风向航母流场浴结构

在舰艄舰载机起飞区域,浴流主要出现在甲板 前端与两侧, 其中处于前端的涡流对舰载机的起飞 影响最大。而在航母后部舰载机降落区, 由于舰岛 的存在, 产生周期性脱落的浴, 并与右侧甲板卷起的 涡相互作用, 严重影响甲板右后方区域,之后其又与 舰艉后方的涡流产生进一步耦合, 在航母尾流区域 形成复杂的浴系。其中, $0^{\circ}$ 风向下浴流主要存在于 右后方甲板及其后方的尾流区; 右舷 $15^{\circ}$ 风向下, 右 侧甲板产生的浴向左翻起作用在舰岛与后方甲板， 受其影响的甲板区域增加; 而在左䑨 $9^{\circ}$ 风向下, 左 侧甲板涡流的强度增大, 而舰岛产生的浴向右偏离 舰载机着舰区域。

\section{4 进舰剖面流场分析}

为了进一步明确舰载机进舰轨迹区域的流场特 征,沿理想进舰轨迹垂直于 $x y$ 平面截取进舰轨迹剖 面,并通过 $Q$ 值云图显示不同风向下该剖面的瞬态 浴结构, 如图 9 所示。图中直线为理想进舰轨迹, 着 舰点位于 $x=210 \mathrm{~m}$ 处。

可以看出, $0^{\circ}$ 风向下甲板后部及尾流中的进舰 运动轨迹区域存在多处浴流, 这将对舰载机着舰产 生不利的影响; 同样在右舷 $15^{\circ}$ 风向下, 浴流向左偏 移, 在舰载机降落区域产生的影响也随之变大; 而在 左舷 $9^{\circ}$ 风向下, 进舰轨迹区域的浴流相对较少, 而 左侧甲板卷起的浴尚未影响到降落区域, 此时有利 于舰载机的安全降落。 

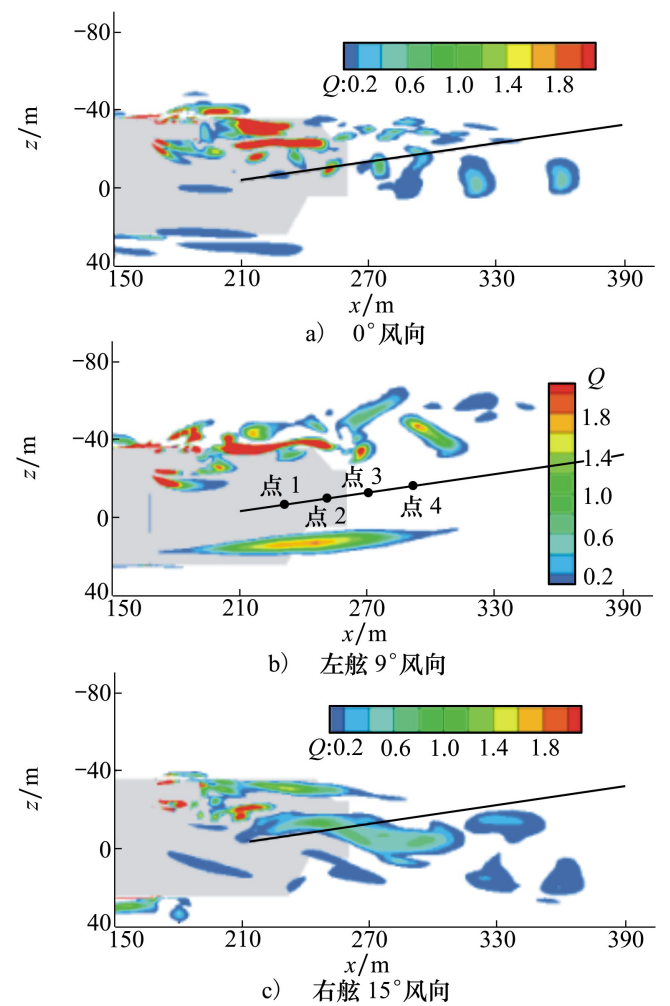

\section{5 进舰轨迹速度分布对比}

图 10 展示了不同风向进舰轨迹上的速度分布 时均值, 从图中可以看出, 左舷 $9^{\circ}$ 风向下速度分布 的波动最小,这也进一步说明了该风向对舰载机的 降落有利; 而在右舷 $15^{\circ}$ 风向下, 速度波动的幅值最 大,其中在接近航母尾部时, 3 个速度分量均产生较 大的变化,此时由于进舰轨迹末端处于舰岛产生涡 流之中, $V_{y}$ 由上洗变为下洗, 对舰载机的安全降落 提出挑战。

\section{$4.60^{\circ}$ 风向进舰轨迹速度波动}

以 $0^{\circ}$ 风向角计算状态为例, 图 11 展示了 $t=48$ $\mathrm{s}, t=49.2 \mathrm{~s}$ 及 $t=50.4 \mathrm{~s} 3$ 种瞬态下理想进舰轨迹上 的速度分布, 并将其同时均结果相比较。结果表明, 速度波动主要存在于着舰前的 $200 \mathrm{~m}$, 即 $x=210 \sim$ $410 \mathrm{~m}$ 范围内; 而瞬态与时均速度分布的差异主要 存在于后部甲板及其临近的尾流区, 尤其是在侧向 和垂向, 大强度的侧风与上下洗将严重影响舰载机 的降落,这是定常计算所不能得到的。

图 9 理想进舰轨迹剖面瞬态 $Q$ 值云图

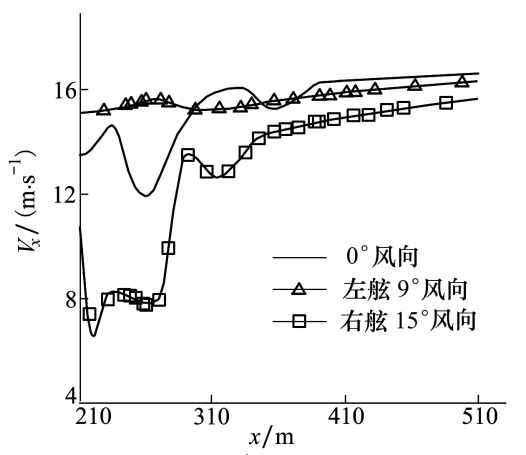

a) $V_{x}$

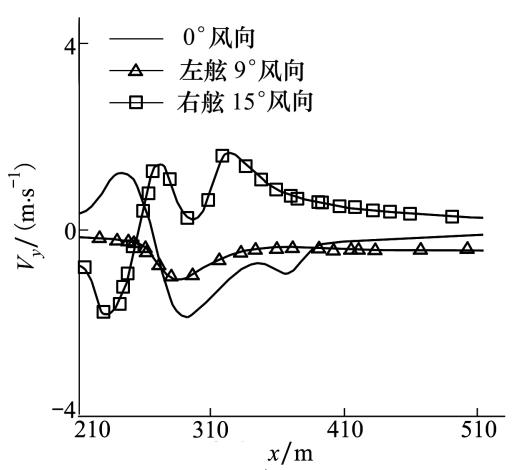

b) $V_{y}$

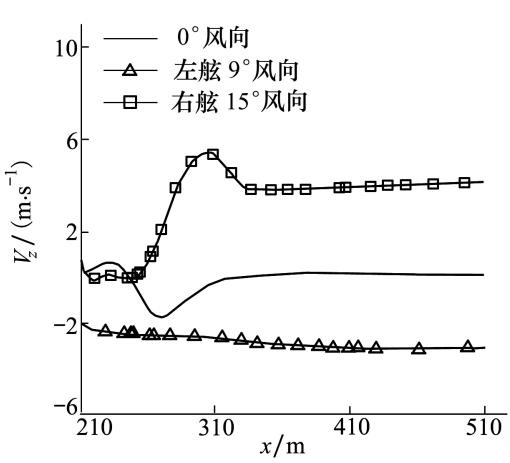

c) $V_{z}$

图 10 理想进舰轨迹上的时均速度分布对比
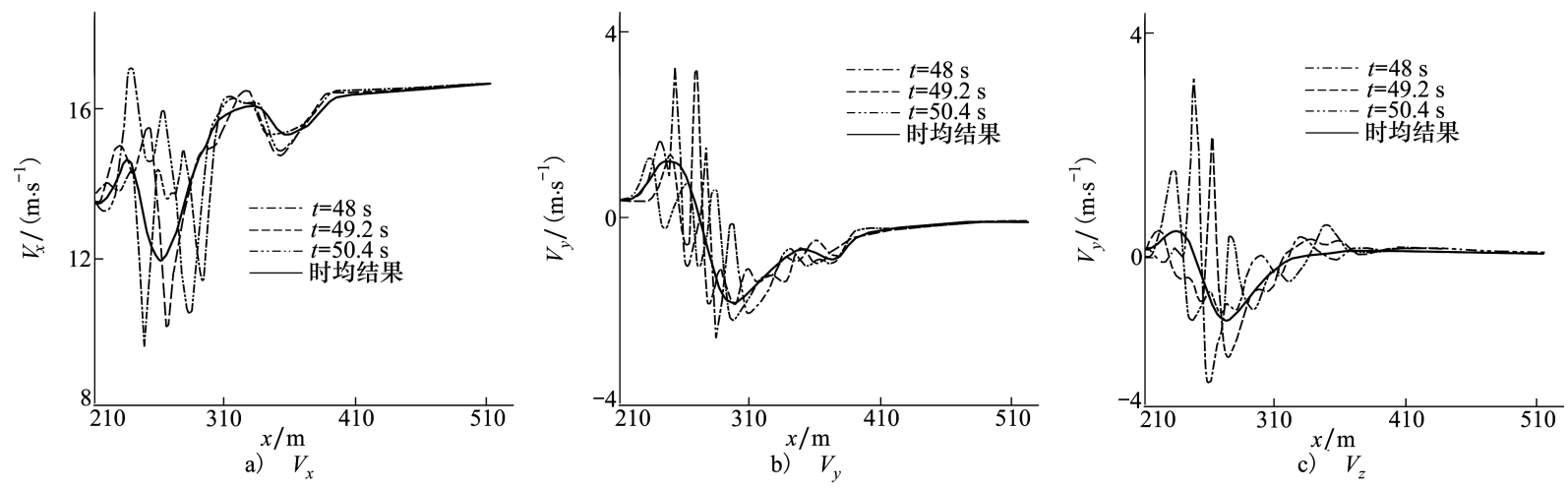

图 11 理想进舰轨迹上不同时刻瞬态速度分布对比 
从图 11 中可以看出, 相比于时均结果, 瞬态速 度的波动主要存在于临近船尾的范围内,在该范围 进舰轨迹上选取 4 个点, 如图 9b) 所示, 于数据采样 平均过程监视其速度变化情况, 得到速度波动范围 如表 1 所示。

表 1 监视点速度波动范围

\begin{tabular}{ccccc}
\hline 位置与速度 & 点 1 & 点 2 & 点 3 & 点 4 \\
\hline$x / \mathrm{m}$ & 230 & 250 & 270 & 290 \\
$V_{x \min } /\left(\mathrm{m} \cdot \mathrm{s}^{-1}\right)$ & 12.583 & 6.382 & 7.009 & 7.086 \\
$V_{x \max } /\left(\mathrm{m} \cdot \mathrm{s}^{-1}\right)$ & 16.845 & 16.669 & 16.051 & 15.423 \\
$V_{y \min } /\left(\mathrm{m} \cdot \mathrm{s}^{-1}\right)$ & -0.682 & -1.767 & -3.082 & -2.836 \\
$V_{y \max } /\left(\mathrm{m} \cdot \mathrm{s}^{-1}\right)$ & 2.348 & 4.504 & 4.031 & 1.439 \\
$V_{z \min } /\left(\mathrm{m} \cdot \mathrm{s}^{-1}\right)$ & -0.725 & -3.243 & -3.243 & -3.842 \\
$V_{z \max } /\left(\mathrm{m} \cdot \mathrm{s}^{-1}\right)$ & 3.181 & 5.071 & 3.793 & 1.574 \\
\hline
\end{tabular}

表 1 表明进舰轨迹上各点速度的大小及方向随 着时间推进而变化。点 $2 、 3$ 接近甲板末端, 3 个方 向速度的波动幅值较其他两点大。而随着远离甲板 末端,进舰轨迹上各点速度波动逐渐放缓。

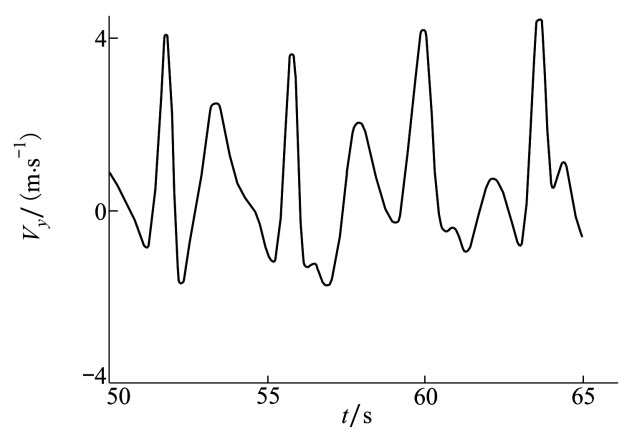

图 12 点 2 处 $V_{y}$ 速度随时间的波动
观察 $t=50 \sim 65 \mathrm{~s}$ 时间段内点 2 处 $V_{y}$ 速度随时 间的变化,如图 12 所示。该状态下速度波动的周期 为 $4 \mathrm{~s}$ 左右, 且存在短时间内上下洗的变化, 这将影 响舰载机的安全降落。

\section{5 结 论}

本文对比了不同数值方法对舰船空气流场的模 拟结果, 对舰船空气流场数值模拟方法及流场特征 进行了探索, 得出的结论如下:

1) 舰船空气流场体现出非稳态特征, 基于 RANS 的非定常计算并不能够模拟出该特征,但基 于 RANS 定常计算得到的流场结构与 DES 方法取 得的时均结果相似。而基于 DES 的数值模拟能够 较好地捕捉舰船空气流场中涡的生成和发展, 其中 基于 $k-\omega \mathrm{SST}$ 湍流模型的 DES-SST 方法所得结果更 为准确;

2) 航母流场中最为突出的是舰岛处周期性脱 落的浴, 及其与甲板边缘、尾部浴流相互作用形成的 复杂浴系。这些浴流在舰载机进舰轨迹上引起较为 强烈的且随着时间推进的速度波动, 导致在航母后 部甲板及其临近的尾流区产生大的侧风及上下洗变 化,其中甲板末端表现最为显著;

3) 不同风向下,航母甲板受浴流影响的区域范 围不同,其中左舷 $9^{\circ}$ 风向下舰载机进舰轨迹区域的 浴流相对较少,且进舰轨迹上的时均速度波动较为 平缓, 对舰载机着舰有利。

\section{参考文献:}

[1] Cheney B, Zan S. CFD Code Validation Data and Flow Topology for TCCP AER-TP-2 Simple Frigate Shape[M]. Canada, National Research Council, 1999

[2] Zan S. Surface Flow Topology for a Simple Frigate Shape[J]. Canadian Aeronautics and Space Journal, 2001, 47( 1) : 33-43

[3] Rajagopalan G, Niazi S, Wadcock A, et al. Experimental and Computational Study of the Interaction between a Tandem-Rotor Helicopter and a Ship [C] // Alexandria, American Helicopter Society 61st Annual Forum, 2005: 729-750

[4] 刘航，赵维义. 直升机载舰空气尾流特性试验方法 $[\mathrm{J}]$. 流体力学实验与测量, 2002, 16(4): 57-63

Liu Hang, Zhao Weiyi. Experimental Methods on Air Wake Characteristics of Shipborne Helicopter $[\mathrm{J}]$. Experiments and Measurements in Fluid Mechanics, 2002, 16(4): 57-63 (in Chinese)

[5] 赵维义, 王占勇. 舰船空气尾流场对直升机着舰的影响研究 $[J]$. 海军航空工程学院学报, 2007, 22(4) : 435-438

Zhao Weiyi, Wang Zhanyong. Research on the Effect of Warship Air-Wake on Helicopter Landing on Shipboard[J] . Journal of Naval Aeronautical Engineering Institute, 2007, 22(4) : 435-438 (in Chinese)

[6] 胡涛, 孙传伟. 舰船空气艉流场试验及仿真技术研究 $[J]$. 直升机技术,2016, 188(2): 11-14 
Hu Tao, Sun Chuanwei. An Experimental and Computational Investigation of Ship Airwake[J]. Helicopter Technique, 2016, 188(2): 11-14 (in Chinese)

[7] Reddy K R, Toffoletto R, Jones K R W. Numerical Simulation of Ship Airwake[J]. Computers \& Fluids, 2000, 29(4): 451-465

[8] Polsky S, Bruner C W. Time-Accurate Computational Simulations of an LHA Ship airwake [ C] // 18th Applied Aerodynamics Conference, 2000: 288-297

[9] 陆超, 姜治芳, 王涛. 利用缩比模型 CFD 数值模拟计算舰船舰面空气流场相似准数的影响探讨 [ J ]. 中国舰船研究, $2008,3(6): 45-48$

Lu Chao, Jiang Zhifang, Wang Tao. Discussion on Comparability of Scaled Models for CFD Numerical Simulation for Ship Airwake $[\mathrm{J}]$. Chinese Journal of Ship Research, 2008, 3(6) : 45-48 (in Chinese)

[10] 陆超, 姜治芳, 王涛. 两种飞行甲板形式的舰船空气流场特性比较 [J]. 舰船科学技术, 2009，31(7)：29-31

Lu Chao, Jiang Zhifang, Wang Tao. A Comparison of Ship's Airwakes with Different Flight Decks[J]. Ship Science and Technology, 2009, 31(7): 29-31 (in Chinese)

[11] 莹亮亮, 魏小辉, 刘成龙, 等. 航母甲板风对舰载机进舰轨迹流场影响分析 $[\mathrm{J}]$. 舰船科学技术, 2014(1):27-30

Ben Liangliang, Wei Xiaohui, Liu Chenglong, et al. Effects of Air Flow on the Landing Track of the Carrier Aircraft [J]. Ship Science and Technology, 2014(1) : 27-30 (in Chinese)

[12] 郜冶, 刘长猛, 贺征. 风向变化产生的航母甲板浴结构特征研究 $[\mathrm{J}]$. 空气动力学学报, 2013, 31(3): 310-315

Gao Ye, Liu Changmeng, He Zheng. Research on CNV Deck Vorties Structure Characteristics Caused by Wind Direction Changes $[\mathrm{J}]$. Acta Aerodynamic Sinca, 2013, 31(3): 310-315 (in Chinese)

[13] 郜冶, 刘长猛. 护卫舰气流场数值计算研究 $[\mathrm{J}]$. 哈尔滨工程大学学报, 2013, 34(5): 599-603

Gao Ye, Liu Changmeng. Numerical Calculation of Frigate Ship Airwake[J]. Journal of Harbin Engineering University, 2013, 34( 5 ) : 599-603 (in Chinese)

[14] 郜冶, 谢辉松. 滑跃起飞过程舰体周围流场的数值模拟 $[\mathrm{J}]$. 空气动力学学报, 2008, 26(4): 513-518

Gao Ye, Xie Huisong. The Numerical Simulation of Flow around the Warship During Ramp Ski-Jump Take-Off [J] . Acta Aerodynamic Sinca, 2008, 26(4) : 513-518 (in Chinese)

[15] Forrest J S, Owen I. An Investigation of Ship Airwakes Using Detached-Eddy Simulation [J]. Computers \& Fluids, 2010,39 (4) : 656-673

[16] 操戈, 程捷, 毕晓波,等. 基于 DES 的舰船空气尾流场特性分析 [J]. 中国舰船研究, 2016, 11(3): 48-54

Cao Ge, Cheng Jie, Bi Xiaobo, et al. Investigation on the Numerical Simulation of Ship Airwake Based on DES [J]. Chinese Journal of Ship Research, 2016, 11(3) : 48-54 (in Chinese)

[17] Spalart P R. Detached-Eddy Simulation[J]. Annual Review of Fluid Mechanics, 2009, 41: 181-202

[18] Strelets M. Detached Eddy Simulation of Massively Separated Flows[ C] // AIAA Fluid Dynamics Conference and Exhibit, 2013: $1-18$

[19] Spalart P R, Deck S, Shur M L, et al. A New Version of Detached-Eddy Simulation, Resistant to Ambiguous Grid Densities[J]. Theoretical and Computational Fluid Dynamics, 2006, 20(3) : 181-195

[20] Spalart P R. Young-Person's Guide to Detached-Eddy Simulation Grids[R]. NASA/CR-2001-211032

[21] Counihan J. Adiabatic Atmospheric Boundary Layers: A Review and Analysis of Data from the Period[J]. Atmos Environ, 1975, $9(10): 871-905$

[22] Hunt J, Wray A, Moin P. Eddies, Stream and Convergence Zones in Turbulent Flows [ R]. N89-24555 Center for Turbulence Research Report, 1988: 193-208 


\title{
Numerical Simulation and Characteristic Analysis of Ship's Air Flow Field
}

\author{
Guo Jiahao $^{1}$, Zhu Xiaoping ${ }^{2}$, Zhou Zhou ${ }^{1}$, Xu Xiaoping ${ }^{1}$
}

(1.School of Aeronautics, Northwestern Polytechnical University, Xi' an 710072, China;

2.Science and Technology on UAV Laboratory, Northwestern Ploytechnical University, Xi'an 710065, China)

\begin{abstract}
The air flow field of ship was simulated by using computational fluid dynamics technology to analyze its prime characteristics with reasonable accuracy. The different results of Reynolds-Averaged Navier-Stokes (RANS) method and Detached Eddy Simulation (DES) were compared, and the calculation traits of these methods were discussed. The results show that the air flow field of ship is unsteady. The accuracy of RANS simulation is insufficient for capturing this unsteady phenomenon. However, DES can catch this with better accuracy and expresses a comparatively great conformity with experimental data. Then, the aircraft carrier's flow field was calculated by DES. The characteristics of vortexes and velocity fluctuation on the ideal landing track were discussed in different wind directions. Those simulations indicate that there are complicated vortexes produced by blunt edges of the island and deck in the flow field. Those vortexes interact and mainly exist in the rear of flight deck and its adjacent air wake. Moreover, they cause a conspicuous and periodical velocity fluctuation on the ideal landing track as time goes on.
\end{abstract}

Keywords : ship air wake; numerical method; DES; aircraft carriers; vortex flow 